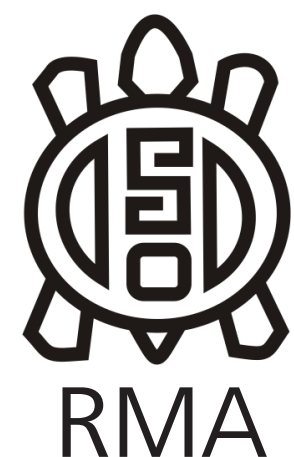

Dossier

\title{
Cambios y continuidades en la movilidad humana a finales del holoceno tardío: cambios entésicos, lesiones articulares e isótopos estables en el sur de Mendoza
}

\author{
Changes and continuities in human mobility at the end of the late \\ holocene: entheseal changes, degenerative joint disease and stable \\ isotopes in southern Mendoza
}

Eva A. Peralta*

*IANIGLA/CONICET Grupo Vinculado San Rafael- Departamento de Antropología, Museo de Historia Natural de San Rafael. E-mail: eperalta@mendoza-conicet.gob.ar

\begin{abstract}
Resumen
La variabilidad en la movilidad humana es uno de los tópicos arqueológicos más abordados en el estudio de las sociedades del pasado. En el sur de la provincia de Mendoza las investigaciones han estudiado la movilidad en el marco de procesos como la introducción de la producción de alimentos y la intensificación en la explotación de los recursos (ca. 2000 años AP). Si bien se ha logrado entender gran parte de esta movilidad a través de los isótopos de $\delta^{18} \mathrm{O}$, es necesario ajustar estos patrones con otros indicadores que puedan observarse a nivel del individuo. En el presente trabajo se articulan estos datos isotópicos con información derivada del análisis de lesiones degenerativas articulares y cambios entésicos que permitan discutir las estrategias de subsistencia y movilidad que implementaron las poblaciones que habitaron la región a finales del Holoceno tardío. Se analizaron muestras osteológicas humanas de diez sitios arqueológicos de la cuenca del Atuel $(n=25)$. Se comparó un entierro múltiple mezclado con entierros aislados e individualizados, que presentan cronologías posteriores a los 2000 años AP. Los patrones de actividad identificados indican grupos que mantienen una alta movilidad para momentos posteriores a la introducción de los cultígenos en el área. Partiendo de un modelo dicotómico ideal (cazadores-recolectores móviles/agricultores sedentarios) pueden entenderse estas tendencias como el resultado de estrategias que combinan a través del tiempo y del espacio, lo productivo y lo extractivo.
\end{abstract}

Palabras clave: Movilidad; estrategias humanas; frontera agrícola; estrés; $\delta^{18} \mathrm{O}$.

\begin{abstract}
Variability in human mobility is one of the most important topics in the study of past societies. In southern Mendoza province, researches deal with this issue in the context of large scale process, such as introduction offood production and intensification in the exploitation of resources (ca. $2000 \mathrm{yr}$ BP). Although mobility can be understood through stable oxygen isotopes $\left(\delta^{18} \mathrm{O}\right)$ we could employ other high resolution proxies, observed at an individual level, to strengthen the evidence. The aim of this work is to articulate isotopic data with degenerative joint diseases and entheseal changes to discuss human strategies about subsistence and mobility that were implemented at the end of Late Holocene. For this purpose, osteological samples of ten archaeological sites from middle Atuel valley $(n=25)$, a place where was discussed the limit of agriculture and intensification process, were studied. We compare isolated versus multiple burials with chronologies after 2000 years BP. Activity patterns point out that high mobility is maintained even after incorporation of cultigens. Considering an ideal dichotomous model (mobile huntergatherers vs sedentary farmers) these tendencies could be understood as a result of a combination of productive and extractive strategies.
\end{abstract}

Keywords: Mobility; human strategies; agricultural frontier; stress; $\delta^{18} \mathrm{O}$.

En el centro occidente argentino, las primeras investigaciones establecieron la frontera agrícola en el sur de Mendoza, en el área asociada a la cuenca del río Atuel (Castro y Tarragó 1992; Lagiglia 1977, 1981). Este territorio se configuró como una zona buffer en la que confluían dos grupos con subsistencias claramente distinguibles: agricultores y cazadores-recolectores, los primeros dominando las latitudes al norte y los segundos al sur (Lagiglia 1977). La introducción de economías productivas se explicaba desde perspectivas históricoculturales a través de un proceso de difusión, por el cual la agricultura era traída por grupos provenientes del norte (Gil 1997-1998; Lagiglia 1982). Este modelo suponía un proceso lineal por el cual los pobladores se 
volvían completamente productores y sedentarios, lo que junto con la innovación tecnológica de la cerámica, implicaba el establecimiento de la vida agrícola hacia los 2000 años AP. El correlato arqueológico sobre el que se apoyaba esta propuesta se basaba principalmente en los macrorestos de cultígenos (i.e. Zea mays, Cucurbita sp., Chenopodium quinoa, Phaseolus vulgaris y Lagenaria sp.), que se hicieron presentes en el registro a partir de esa fecha (Gil 1997-1998, 2003; Lagiglia 1977, 1981). A continuación de este período de agricultores (pero sin fecha cierta) se habría sucedido una nueva etapa, caracterizada por el reingreso de cazadores-recolectores de raigambre mapuche, con los que finalmente se habrían encontrado los europeos a su arribo (Semper y Lagiglia 1962-1968).

El estado de las investigaciones en la zona de estudio señala un cambio importante en el registro arqueológico hacia los 2000 años AP (Giardina et al. 2015; Gil et al. 2006, 2010, 2012; Gil y Neme 2010; Neme 2007; Neme y Gil 2008, 2012). Las evidencias disponibles definen al valle del Atuel como un área transicional y de interacción en la que confluyen diversas estrategias de subsistencia y movilidad. Parte de estas estrategias se han explicado en asociación a la incorporación de producción de alimentos y/o intensificación en la explotación de recursos, procesos que suponen una reducción en la movilidad respecto de períodos anteriores (Gil y Neme 2012; Neme 2007; Neme y Gil 2008). Si bien se han evaluado reducciones en la movilidad que permitan dar cuenta de un cambio de estrategia de subsistencia, se requieren exploraciones a escala del individuo que proponemos abordar desde los cambios entésicos y lesiones degenerativas articulares, bajo el supuesto de que pueden ser informativas del esfuerzo físico desarrollado por el individuo si existe un control de la variabilidad asociada al sexo y la edad.

El objetivo de este trabajo es realizar un aporte a los estudios sobre las estrategias de subsistencia y movilidad que implementaron las poblaciones que habitaron la región a finales del Holoceno tardío, específicamente en relación al proceso de incorporación de la agricultura. Se articulan, en una aproximación inicial, análisis de cambios entésicos y lesiones articulares con información isotópica para discutir cambios en la movilidad y problematizar las interpretaciones basadas en los extremos cazarecolección/agricultura.

\section{Marcadores óseos de actividad y movilidad}

El estudio de las actividades cotidianas se ha abordado empleando diferentes marcadores a nivel óseo. Entre los más utilizados se encuentran las lesiones asociadas a la enfermedad degenerativa articular u osteoartrosis, y los cambios entésicos o marcadores de estrés músculoesqueletal (Hawkey y Merbs 1995; Robb 1998; Capasso et al. 1999; Jurmain et al. 2012; Kennedy 1989; Santos et al. 2011; Villotte et al. 2010a, 2010b; Villotte y Knüsel 2013). Basándose en las propiedades plásticas del hueso, estos marcadores se manifestarían como producto de las continuas exigencias mecánicas requeridas durante la realización de actividades pautadas y rutinarias (Robson Brown et al. 2008; Galtés et al. 2007; Kennedy 1989; Rodrigues Carvalho 2004). En los comienzos de su aplicación fuera del campo médico y forense, se utilizaban para especificar algún tipo de actividad puntual (Capasso at al. 1999; Jurmain 1977; Mendonça de Souza 1992). Dada la gran variedad de movimientos asociados a una misma actividad y la gran cantidad de actividades que requieren de un mismo movimiento, en los últimos tiempos se ha buscado separarse de esta tendencia. Asimismo la etiología multifactorial de los marcadores óseos implica que no todos son consecuencia de la realización de actividades rutinarias y/o intensivas (Jurmain et al. 2012; Santos et al. 2011; Weiss y Jurmain 2007). Entre las aplicaciones actuales en arqueología se han llevado a cabo estos estudios desde perspectivas poblacionales para discutir y definir patrones de actividad asociados con la organización del trabajo y el tipo de subsistencia (Bridges 1989, 1992, 1994; Cope et al. 2005; Henderson et al. 2013a; 2013b; Quevedo 2000; Villotte et al. 2010b; Wilczak 1998). Distintos trabajos han interpretado estos marcadores óseos como informativos sobre la movilidad, principalmente entre grupos cazadores-recolectores (Quevedo 2000; Salega y Fabra 2013; Scabuzzo 2012; Al-Oumaoui et al. 2004). Al mismo tiempo, trabajos que exceden los estudios de estrés ocupacional han correlacionado las estrategias de subsistencia con las de movilidad. En muchos casos se ha asumido que en contextos productivos las poblaciones humanas tienden a un proceso de sedentarización, mientras que las economías extractivas se asociarían con una alta movilidad. Partiendo de estos contextos extremos, se han desarrollado distintas alternativas y diversas combinaciones de movilidad y subsistencia.

\section{Isótopos estables y movilidad}

Las aplicaciones de los isótopos de oxígeno $\left({ }^{18} \mathrm{O} /{ }^{16} \mathrm{O}\right)$ en arqueología se concentraron en temáticas relacionadas con la movilidad y procedencia geográfica (Knudson 2009; Sponheimer y Lee-Thorp 1999; Turner et al. 2009). Estos isótopos se presentan en los carbonatos y fosfatos de la hidroxiapatita del hueso y del esmalte dental, y reflejan principalmente la composición isotópica del agua corporal. A su vez, estos valores dependen del agua ingerida, y en menor medida del oxígeno presente en el aire y en los recursos alimenticios (Sponheimer y LeeThorp 1999; Turner et al. 2009; White et al. 2004). Al mismo tiempo, el agua ingerida presenta una variabilidad regular en relación con la latitud, la altitud, la aridez, la estacionalidad y el régimen de precipitaciones, factores relacionados con los ciclos de evaporación y precipitación que resultan en el empobrecimiento o enriquecimiento de los valores del isótopo pesado $\left({ }^{18} \mathrm{O}\right)$ en el agua (Dansgaard 1964; Gat 1996; White et al. 2004).

Para el sur de Mendoza se cuenta con una base de 
datos de $\delta^{18} \mathrm{O}$ tanto de fuentes de agua como de restos óseos humanos de diferentes sitios arqueológicos (Gil et al. 2012, Ugan et al. 2012). Algunos de estos datos disponibles serán rediscutidos en este trabajo, junto con los cambios entésicos y lesiones asociadas a osteoartrosis.

\section{Hipótesis y Expectativas}

Como hipótesis de este trabajo proponemos que hacia los 2000 años AP los grupos humanos del sur de Mendoza optaron por una estrategia de producción de alimentos asociada con una reducción en la movilidad. Dado que el sur de Mendoza estuvo habitado por cazadoresrecolectores que pudieron o no incorporar agricultura, uno de los supuestos derivados de nuestra hipótesis es que al variar su estrategia hacia la producción de alimentos, y dados los procesos de saturación del espacio e intensificación que estarían ocurriendo para ese período, su movilidad se habría visto restringida, tendiendo a una mayor permanencia en determinados lugares más aptos para las actividades agrícolas. En términos de marcadores óseos, esperamos que esta reducción en la movilidad se vea reflejada en prevalencias bajas de cambios entésicos y lesiones asociadas a osteoartrosis en los miembros inferiores con respecto a los superiores, ya que se encuentran más expuestos al estrés producto de la locomoción y en consecuencia a las variaciones en la misma (Capasso et al 1999; Jurmain 1977; Neves 1984). En cuanto a los valores de $\delta^{18} \mathrm{O}$, se espera identificar poca variabilidad interindividual y una correspondencia con los valores de las fuentes de aguas más próximas a los sitios de donde provienen las muestras.

En este trabajo proponemos caracterizar los patrones de desgaste articular y de cambios entésicos de distintos conjuntos óseos humanos del sur de Mendoza, interpretando sus implicancias en términos de movilidad y subsistencia. Asimismo vinculamos esta información sobre movilidad con la que aportan los isótopos de oxígeno.

\section{Materiales y Métodos}

La muestra analizada comprende sitios arqueológicos ubicados en la cuenca media del valle del Atuel (Tabla
1, Figura1). El valle del Atuel presenta una mayor concentración de sitios con entierros humanos con respecto a otras áreas del sur de Mendoza. Dentro de esta cuenca existen tanto entierros aislados como grandes áreas formales de entierro con un gran número de individuos. Cerro Negro-1 (CN-1), Puesto Médano Aisol (PMA), Arroyo Los Jilgueros (ALJ), Zanjón Los Jilgueros (ZLJ), Camping Familiar Cristiano (CFC-1), La Olla (LO), Valle Grande (VG), Cañón del Atuel (CA) son sitios que presentan sólo un individuo, Rincón del Atuel-1 (RA-1) presenta 4 y Cañada Seca-1 (CS-1) tiene un NMI de 23 (Peralta 2015). Para desarrollar el análisis se decidió agrupar aquellos sitios que pudieran caracterizarse como entierros aislados, separándolos de CS-1, por sus características claramente diferenciables en cuanto al tamaño muestral y a la modalidad de entierro. De esta forma se comparan sitios con modalidades de entierro diferentes, posteriores al momento del registro de los primeros cultígenos. En la Tabla 1 se detallan los sitios considerados con los fechados y la información isotópica disponible.

Para la determinación del sexo se utilizaron los rasgos morfológicos de la pelvis y del cráneo. Para la pelvis se consideraron la escotadura ciática, el arco ventral, la concavidad subpúbica, el aspecto medial de la rama isquiopúbica, el criterio del arco y el ángulo subpúbico (Bruzek 2002; Buikstra y Ubelaker 1994). Para el cráneo se observó la glabela, proceso mastoides, margen supraorbital, eminencia del mentón y cresta nucal (Buikstra y Ubelaker 1994). Para la estimación de la edad en los casos en que se contaba con coxales asociados al resto del postcráneo, se usaron los rasgos de la sínfisis púbica y superficie auricular (Todd 1920; Lovejoy et al. 1985; Meindl y Lovejoy 1989; Buikstra y Ubelaker 1994). Se emplearon las categorías de Adulto joven (20-35 años), Adulto medio (35-50 años) y Adulto maduro (>50 años) de acuerdo con Buikstra y Ubelaker (1994). Para el entierro secundario múltiple (CS-1) se diagnosticaron 13 hemipelvis y tres cráneos completos para estimar sexo y edad, aunque no fue posible asociarlos con otros elementos. En este caso solo se utilizó la categoría Adulto ya que no fue posible asociar los elementos diagnósticos (coxales y cráneo) con los elementos del postcráneo.

Figura 1. Sitios mencionados en el texto: Cerro Negro-1 (CN-1), Puesto Médano Aisol (PMA), Arroyo Los Jilgueros (ALJ), Zanjón Los Jilgueros (ZLJ), Camping Familiar Cristiano (CFC-1), La Olla (LO), Valle Grande (VG), Cañón del Atuel (CA), Rincón del Atuel-1 (RA-1) y Cañada Seca-1 (CS-1). Modificado de Giardina et al. 2015.

Figure 1. Archaeological sites mentioned in the text: Cerro Negro-1 (CN-1), Puesto Médano Aisol (PMA), Arroyo Los Jilgueros $(A L J)$, Zanjón Los Jilgueros (ZL), Camping Familiar Cristiano (CFC-1), La Olla (LO), Valle Grande (VG), Cañón del Atuel (CA), Rincón del Atuel-1 (RA-1) and Cañada Seca-1 (CS-1). Modified from Giardina et al. 2015.

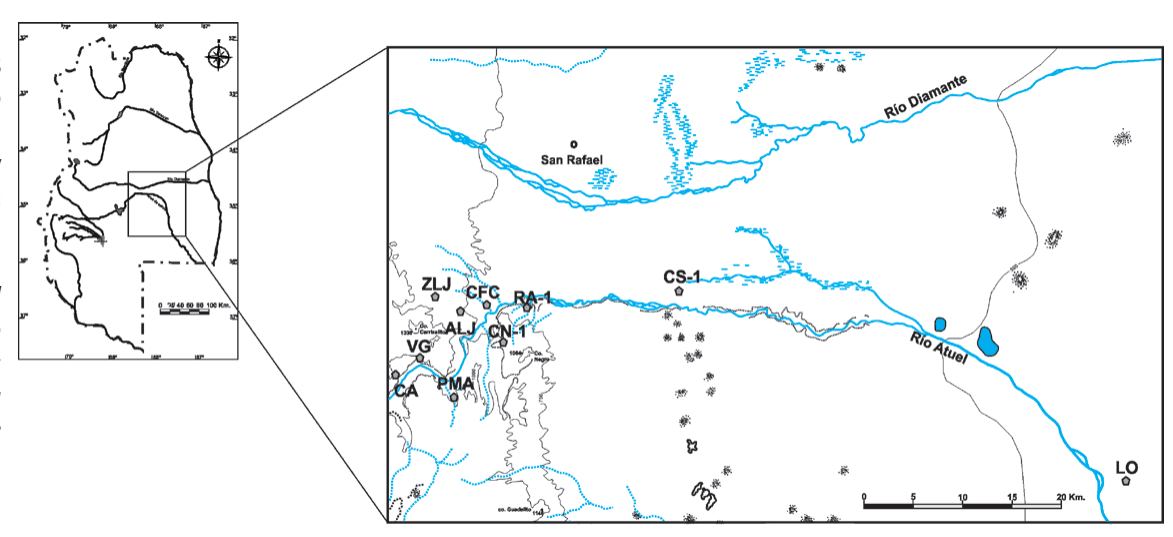




\begin{tabular}{|c|c|c|c|c|c|c|c|}
\hline Sitio & NMI & $\begin{array}{c}\text { NMI } \\
\text { analizado }\end{array}$ & Fecha directa & Isótopos & Desierto & Sexo & Categoría etaria \\
\hline Ao. Los Jilgueros (ALJ) & 1 & 1 & - & $\begin{array}{c}\delta^{15} \mathrm{~N} \\
\delta^{13} \mathrm{C}_{\mathrm{col},} \\
\delta^{13} \mathrm{C}_{\mathrm{apa},} \\
\delta^{18} \mathrm{O}\end{array}$ & MONTE & M & AdJ \\
\hline Cañada Seca-1 (CS-1) & 23 & 13 & $\begin{array}{l}1790 \pm 50 \\
1420 \pm 60\end{array}$ & $\begin{array}{c}\delta^{15} \mathrm{~N} \\
\delta^{13} \mathrm{C}_{\mathrm{col}} \\
\delta^{13} \mathrm{C}_{\text {apar }} \\
\delta^{18} \mathrm{O} \\
\delta^{15} \mathrm{~N}\end{array}$ & MONTE & $\begin{array}{c}M, M, M \\
M, M, M \\
M, F\end{array}$ & AdM \\
\hline Cerro Negro-1 (CN-1) & 1 & 1 & $580 \pm 35$ & $\begin{array}{c}\delta^{13} \mathrm{C}_{\mathrm{col},} \\
\delta^{13} \mathrm{C}_{\text {apal }} \\
\delta^{18} \mathrm{O} \\
\delta^{15} \mathrm{~N}\end{array}$ & MONTE & M & AdM \\
\hline La Olla (LO) & 1 & 1 & $645 \pm 42$ & $\begin{array}{l}\delta^{13} C_{\mathrm{col}} \\
\delta^{13} \mathrm{C}_{\mathrm{apa}} \\
\delta^{18} \mathrm{O}\end{array}$ & MONTE & I & $\mathrm{AdJ}$ \\
\hline Puesto Médano Aisol (PMA) & 1 & 1 & - & $\begin{array}{c}\delta 13 \mathrm{~N}, \\
\delta^{13} \mathrm{C}_{\mathrm{col}} \\
\delta^{13} \mathrm{C}_{\mathrm{apa}} \\
\delta^{18} \mathrm{O}\end{array}$ & MONTE & M & AdJ \\
\hline Rincón del Atuel-1 (RA-1) & 4 & 4 & $1760 \pm 70$ & $\begin{array}{c}\delta^{13} \mathrm{~N}_{1} \\
\delta^{13} \mathrm{C}_{\mathrm{col}} \\
\delta^{13} \mathrm{C}_{\mathrm{apa}} \\
\delta^{18 \mathrm{O}} \mathrm{O}\end{array}$ & MONTE & $M, M, M, F$ & AdMa/AdJ \\
\hline Valle Grande (VG) & 1 & 1 & - & - & MONTE & M & AdM \\
\hline Camping Familiar Cristiano (CFC-1) & 1 & 1 & $1094 \pm 35$ & $\begin{array}{c}\delta^{13} C_{\text {apa }} \\
\delta^{18} \mathrm{O}\end{array}$ & MONTE & M & AdMa \\
\hline Zanjón Los Jilgueros (Zப) & 1 & 1 & - & - & MONTE & M & AdJ \\
\hline Cañón del Atuel (CA) & 1 & 1 & - & $\begin{array}{c}\delta^{15} \mathrm{~N}, \\
\delta^{13} \mathrm{C}_{\mathrm{col}} \\
\delta^{13} \mathrm{C}_{\mathrm{apa}} \\
\delta^{18} \mathrm{O}\end{array}$ & MONTE & $\mathrm{M}$ & AdM \\
\hline
\end{tabular}

Tabla 1. Sitios analizados en este trabajo. Referencias: $M=$ Masculino, F=Femenino, AdJ=Adulto Joven, AdM=Adulto medio, $\mathrm{AdMa}=$ Adulto maduro, $\mathrm{Ad}=$ Adulto

Table 1. Sites analyzed in this work. References: $M=$ Male, $F=$ Female, Adj=Young adult, AdM=Middle adult, AdMa $=$ Old Adult, Ad=Adult.

estos cuadrantes se le asignó un valor entre 0 y 3 de acuerdo al grado de manifestación del desgaste articular. La gradación utilizada para las proliferaciones periarticulares fue la siguiente: 0) ninguna indicación de osteoartrosis; 1) se evidencian irregularidades incipientes en la zona periarticular, menores a $1 \mathrm{~mm}$ de longitud; 2) los osteofitos son claramente visualizables y no exceden los cinco $\mathrm{mm}$ de longitud; 3) las excrecencias
Se registraron los cambios entésicos y los procesos de degeneración articular exclusivamente sobre los huesos largos del esqueleto apendicular. Este recorte se realizó para garantizar la comparación entre muestras con diferentes estados de conservación y optimizar el tiempo de relevamiento. En estudios subsiguientes se incluirán elementos del esqueleto axial. El análisis se realizó a nivel del elemento, agrupando los entierros aislados por un lado y los individuos de CS-1 por otro. En el caso de las lesiones articulares, se calcularon los porcentajes por articulación (hombro-codo-muñeca) de afectados y no afectados. En el caso de los cambios entésicos, se calcularon los porcentajes de prevalencia de todos los rasgos para cada entesis. Las comparaciones se realizaron entre ambos grupos separando miembros superiores de miembros inferiores.

Se siguió el método Coimbra (Henderson et al. 2013a, 2016) para el estudio de los cambios entésicos, por el cual cada área de inserción se dividió en dos zonas, registrándose la formación ósea (FO1) y la erosión (ERO1) en la zona 1 y el cambio textural (CT), la formación de hueso (FO), la erosión (ERO), la porosidad fina (POF), la macro-porosidad (MPO) y la presencia de cavidades (CA) en la zona 2. La zona 1 refiere al margen opuesto al ángulo agudo en el cual las fibras se insertan. La zona 2 comprende el margen y la superficie restantes. Exceptuando al CT, para el que solo se consideraron dos categorías (0 y 1), las restantes variables se registraron considerando tres categorías ( 0 a 2 ) de acuerdo con su grado de manifestación (Henderson et al. 2013a, 2016).

Para el relevamiento de las lesiones articulares se usaron las recomendaciones presentes en el trabajo de Luna et al. (2017), dividiéndose la articulación entre borde y superficie interna y a su vez en cuatro cuadrantes. A cada uno de tienen una longitud que excede los cinco $\mathrm{mm}$, lo que se corresponde con una afección grave de la articulación. Se incluyen esta la eburnación y anquilosis. Para la porosidad se siguió la siguiente categorización: 0) sin porosidad; 1) menos de un tercio de la superficie afectada; 2) entre el un tercio y dos tercios; 3) más de dos tercios. Para cada articulación, los valores asignados para cada cuadrante se sumaron. El resultado se dividió por la cantidad de cuadrantes efectivamente relevados, de manera de corregir los valores finales y asegurar comparaciones adecuadas en los porcentajes de bordes y superficies afectados (Luna et al. 2017).

Las superficies articulares analizadas fueron las correspondientes al húmero, radio, cúbito, fémur y tibia. En CS-1 se analizó un total de 121 superficies articulares de miembros superiores y 92 de miembros inferiores. Los entierros aislados se agruparon en una única categoría, alcanzando un total de 41 superficies articulares de miembros superiores y 32 de miembros inferiores. Sobre estas cantidades se estimaron las prevalencias de cambios o lesiones por articulación, separando los miembros superiores de los inferiores. Las inserciones musculares analizadas fueron las correspondientes a los músculos Bíceps, Brachialis, Infraspinatius, Supraspinatus, Subscapularis, Teres minor, Tríceps, Gluteus medius, Gluteus minimus, Iliopsoas, Triceps surae, además del origen del Common extensor y Common flexor (Cantarella 1999, Cardoso y Henderson 2013; Henderson et al. 2013a, 2013b). En CS-1 se analizaron 161 éntesis de miembros superiores y 73 de miembros inferiores. En el conjunto de entierros aislados se analizaron 53 éntesis de miembros superiores y 24 de miembros inferiores. El cálculo de las prevalencias se realizó por entesis para todos los rasgos, separando miembros superiores e inferiores. Para evaluar diferencias significativas se aplicó 
Figura 2. Prevalencias de cambios entésicos en miembros superiores e inferiores de CS-1 y entierros aislados. Referencias: CS-MS (Cañada Seca-Miembros Superiores), EA-MS (Entierros Aislados-Miembros Superiores), CS-MI (Cañada Seca-Miembros Inferiores), EA-MI (Entierros Aislados-Miembros Inferiores), FO1 (Formación Ósea en zona 1), ERO1 (Erosión en zona 1), CT (Cambio Textural), FO (Formación Ósea), ERO (Erosión), POF (Porosidad Fina), MPO (Macroporosidad), CA (Cavidades).

Figure 2. Entheseal changes prevalence in lower and upper member limbs from CS-1 and isolated burials. References: CS-MS (Cañada Seca-Upper Limbs), EA-MS (Isolated burials-Upper Limbs), CS-MI (Cañada Seca-Lower Limbs), EA-MI (Isolated burials-Lower Limbs), FO1 (Bone formation, zone 1), ERO1 (Erosion, zone 1), CT (Textural change), FO (Bone formation), ERO (Erosion), POF (Fine porosity), MPO

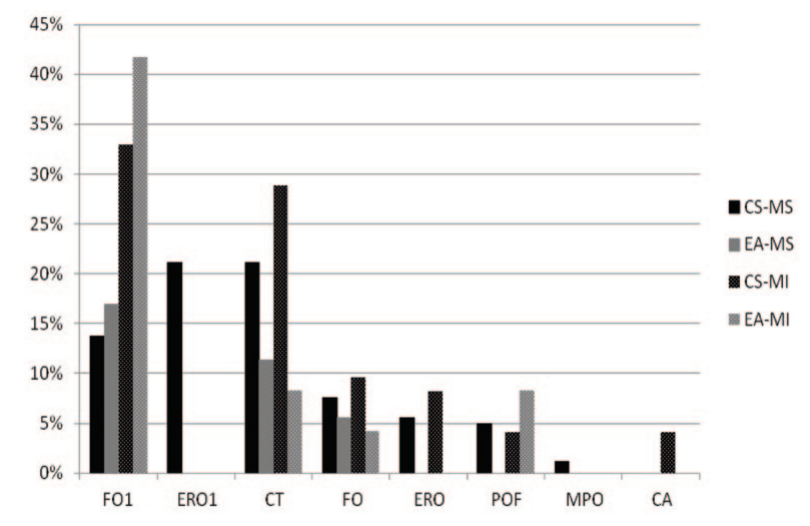
(Macroporosity), CA (Cavitation).

el test $X^{2}$ con un límite de $\mathrm{p} \leq 0.05$, empleando el software PAST 2.05 (Hammer et al. 2001).

\section{Resultados}

\section{Lesiones articulares}

En CS-1 no se observaron diferencias significativas entre miembros superiores e inferiores en el borde $\left(X^{2}=1.2899\right.$; $p=0.25600)$, pero sí en la superficie articular afectada $\left(X^{2}=4.1674 ; p=0.041209\right) ;$ los miembros superiores se mostraron más afectados que los inferiores. A nivel de la articulación, el codo presentó una mayor prevalencia de degeneración articular, junto con la rodilla y el tobillo, tanto en borde como en superficie (Tabla 2). En los entierros aislados no se observaron diferencias significativas entre miembros superiores e inferiores $\left(X^{2}=0.46405 ; p=0.49574\right)$. Asimismo las articulaciones más afectadas fueron el codo, la rodilla y el tobillo, al igual que en CS-1 (Tabla 2). Para estos sitios, se pudo estimar la edad de los individuos, y no se encontró que el desgaste fuera mayor en los individuos de mayor edad. En comparación con los entierros aislados, CS-1 presenta mayores prevalencias de cambios articulares, tanto en el borde como en la superficie (Tabla 2), siendo significativa la diferencia para los miembros superiores $\left(X^{2}=4.9625 ; p=0.025902\right)$ pero no para los inferiores $\left(X^{2}=3.7629 ; p=0.052402\right)$.

\begin{tabular}{|c|c|c|c|c|c|c|c|}
\hline \multirow{2}{*}{ Sitio } & \multirow{2}{*}{ Articulación } & \multicolumn{3}{|c|}{ Borde } & \multicolumn{3}{|c|}{ Superficie } \\
\hline & & Afectado & No afectado & Total general & Afectado & No afectado & Total general \\
\hline \multirow{19}{*}{ CS-1 } & Codo & 50,0 & 50,0 & 100,0 & 15,2 & 84,8 & 100,0 \\
\hline & D & 59,4 & 40,6 & 100,0 & 12,5 & 87,5 & 100,0 \\
\hline & 1 & 41,2 & 58,8 & 100,0 & 17,6 & 82,4 & 100,0 \\
\hline & Hombro & 31,6 & 68,4 & 100,0 & 21,1 & 78,9 & 100,0 \\
\hline & D & 40,0 & 60,0 & 100,0 & 20,0 & 80,0 & 100,0 \\
\hline & 1 & 22,2 & 77,8 & 100,0 & 22,2 & 77,8 & 100,0 \\
\hline & Muñeca & 30,6 & 69,4 & 100,0 & 2,8 & 97,2 & 100,0 \\
\hline & D & 47,1 & 52,9 & 100,0 & 0,0 & 100,0 & 100,0 \\
\hline & 1 & 15,8 & 84,2 & 100,0 & 5,3 & 94,7 & 100,0 \\
\hline & Total general & 41,3 & 58,7 & 100,0 & 12,4 & 87,6 & 100,0 \\
\hline & Cadera & 0,0 & 100,0 & 100,0 & 3,4 & 96,6 & 100,0 \\
\hline & $\mathrm{D}$ & 0,0 & 100,0 & 100,0 & 7,1 & 92,9 & 100,0 \\
\hline & 1 & 0,0 & 100,0 & 100,0 & 0,0 & 100,0 & 100,0 \\
\hline & Rodilla & 45,0 & 55,0 & 100,0 & 7,5 & 92,5 & 100,0 \\
\hline & $\mathrm{D}$ & 40,9 & 59,1 & 100,0 & 0,0 & 100,0 & 100,0 \\
\hline & 1 & 50,0 & 50,0 & 100,0 & 16,7 & 83,3 & 100,0 \\
\hline & Tobillo & 56,5 & 43,5 & 100,0 & 0,0 & 100,0 & 100,0 \\
\hline & D & 66,7 & 33,3 & 100,0 & 0,0 & 100,0 & 100,0 \\
\hline & 1 & 45,5 & 54,5 & 100,0 & 0,0 & 100,0 & 100,0 \\
\hline \multirow{21}{*}{ EA } & Total general & 33,7 & 66,3 & 100,0 & 4,3 & 95,7 & 100,0 \\
\hline & Codo & 34,8 & 65,2 & 100,0 & 0,0 & 100,0 & 100,0 \\
\hline & $\mathrm{D}$ & 45,5 & 54,5 & 100,0 & 0,0 & 100,0 & 100,0 \\
\hline & 1 & 25,0 & 75,0 & 100,0 & 0,0 & 100,0 & 100,0 \\
\hline & Hombro & 0,0 & 100,0 & 100,0 & 0,0 & 100,0 & 100,0 \\
\hline & D & 0,0 & 100,0 & 100,0 & 0,0 & 100,0 & 100,0 \\
\hline & 1 & 0,0 & 100,0 & 100,0 & 0,0 & 100,0 & 100,0 \\
\hline & Muñeca & 13,3 & 86,7 & 100,0 & 0,0 & 100,0 & 100,0 \\
\hline & D & 14,3 & 85,7 & 100,0 & 0,0 & 100,0 & 100,0 \\
\hline & 1 & 12,5 & 87,5 & 100,0 & 0,0 & 100,0 & 100,0 \\
\hline & Total general & 23,8 & 76,2 & 100,0 & 0,0 & 100,0 & 100,0 \\
\hline & Cadera & 0,0 & 100,0 & 100,0 & 0,0 & 100,0 & 100,0 \\
\hline & D & 0,0 & 100,0 & 100,0 & 0,0 & 100,0 & 100,0 \\
\hline & 1 & 0,0 & 100,0 & 100,0 & 0,0 & 100,0 & 100,0 \\
\hline & Rodilla & 18,8 & 81,3 & 100,0 & 0,0 & 100,0 & 100,0 \\
\hline & D & 12,5 & 87,5 & 100,0 & 0,0 & 100,0 & 100,0 \\
\hline & 1 & 25,0 & 75,0 & 100,0 & 0,0 & 100,0 & 100,0 \\
\hline & Tobillo & 25,0 & 75,0 & 100,0 & 0,0 & 100,0 & 100,0 \\
\hline & D & 25,0 & 75,0 & 100,0 & 0,0 & 100,0 & 100,0 \\
\hline & 1 & 25,0 & 75,0 & 100,0 & 0,0 & 100,0 & 100,0 \\
\hline & Total general & 15,6 & 84,4 & 100,0 & 0,0 & 100,0 & 100,0 \\
\hline
\end{tabular}

Tabla 2. Prevalencias de lesiones articulares en miembros superiores e inferiores de CS-1 y EA (entierros aislados).

Table 2. Degenerative joint diseases prevalence in lower and upper member limbs from CS-1 and EA (isolated burials).

\section{Cambios entésicos}

Los miembros superiores se mostraron en general menos afectados que los inferiores tanto en CS-1 como en los entierros aislados (Figura 2). Esta tendencia se observó particularmente en el rasgo FO1 que presentó diferencias significativas, tanto en CS-1 $\left(X^{2}=11.738 ; p=0.000612\right)$ como en los entierros aislados $\left(X^{2}=5.416\right.$; $\mathrm{p}=0.019953)$. En CS-1 los miembros superiores se vieron más afectados solo en ERO1, POF y MPO (Figura 2), aunque fue significativa la diferencia solamente en ERO1 ( $\left.X^{2}=18.037 ; p=2.1667 E-05\right)$. En este sitio aunque todas las éntesis presentaron algún tipo de cambio, las inserciones del bíceps y el brachialis son las que presentaron mayores frecuencias (Figura 3). En los entierros aislados, los miembros superiores se mostraron un poco más intensamente afectados en CT y FO, sin diferencias significativas (CT: $X^{2}=0.15835 ; p=0.69068 ; F O$ : $X^{2}=0.074839 ; p=0.78442$ ) (Figura 2). En este conjunto, el origen del extensor y las inserciones del bíceps, del tríceps y del brachialis fueron las únicas que presentaron cambios entésicos (Figura 3). 


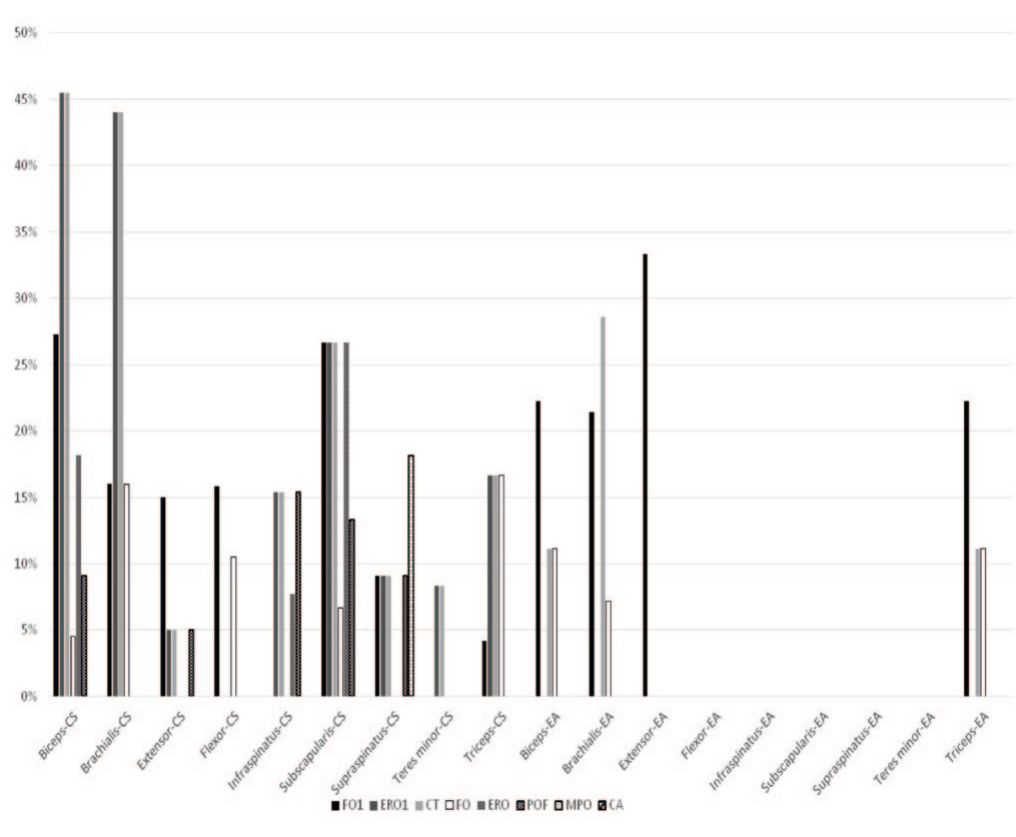

Figura 3. Cambios entésicos en miembros superiores para cada inserción. Referencias: $\mathrm{CS}=$ Cañada Seca 1, EA=entierros aislados.

Figure 3. Entheseal changes in upper limbs for each insertion. References: $\mathrm{CS}=$ Cañada Seca-1, $E A=i s o l a t e d$ burials.

combinado de ambas. En los entierros aislados se observa una dispersión similar a la de CS-1. En este grupo, los individuos de RA-1, CN-1 y CFC-1, se asocian con los valores de NevadoPayunia, mientras que los de ALJ, PMA y LO presentan valores más negativos y vinculados al Atuel Medio.

\section{Discusión}

Este trabajo se propuso abordar de forma preliminar la integración de líneas de evidencia asociadas con la movilidad humana que aportaran al conocimiento

Comparando ambos conjuntos, CS-1 presentó prevalencias mayores que los entierros aislados para casi todos los rasgos, exceptuando FO1 en miembros superiores e inferiores y POF en miembros inferiores (Figura 2). Esta diferencia fue significativa en el caso de la ERO1 en miembros superiores $\left(X^{2}=13.307 ; p=0.000264\right)$ y el $C T$ en miembros inferiores $\left(X^{2}=4.1691 ; p=0.041167\right)$.

\section{$\delta^{18} \mathrm{O}$}

En la Figura 4 se graficaron los valores promedio de $\delta^{18} \mathrm{O}$ de las aguas superficiales según la región geográfica (Gil et al. 2012; Ugan et al. 2012) y los valores individuales obtenidos de las muestras humanas. Las aguas de Alta Cordillera y Piedemonte presentan valores de $\delta^{18} \mathrm{O}$ que varían entre $-20 \%$ y $-12 \%$, las de Atuel Medio oscilan entre $-14 \%$ y $-13 \%$, y las de Nevado-Payunia se encuentran entre $-9 \%$ y $-4 \%$ (Ugan et al. 2012). Los valores de CS- 1 se encuentran entre $-12 \%$ y $-5 \%$, y podrían explicarse por el uso de las aguas de NevadoPayunia y de Atuel Medio. Existe una concentración de individuos entre las mismas que podría responder a un uso de la relación subsistencia/movilidad en un contexto en el cual se habrían incorporado estrategias productivas en una economía tradicionalmente extractiva. Los patrones de cambios entésicos no fueron concordantes con la expectativa de encontrar menores señales de estrés en miembros inferiores en relación a los superiores. Ambos miembros se comportaron de manera muy similar en algunos de los rasgos asociados a cambios entésicos, y en otros casos los miembros inferiores mostraron más cantidad de indicadores de estrés mecánico que los superiores. Solamente se vieron más afectados los miembros superiores de manera significativa en ERO1 en la muestra de CS-1. Por otra parte, los patrones de lesiones articulares asociadas a osteoartrosis tampoco mostraron concordancia con lo esperado, ya que los miembros inferiores solo estuvieron menos expuestos al deterioro articular que los superiores en CS-1 en el interior de las superficies articulares de y no a nivel periarticular. El codo y el tobillo fueron las articulaciones más afectadas. El desgaste articular en el codo se relacionaría tanto con actividades de procesamiento de alimentos como de caza, por lo que este patrón podría

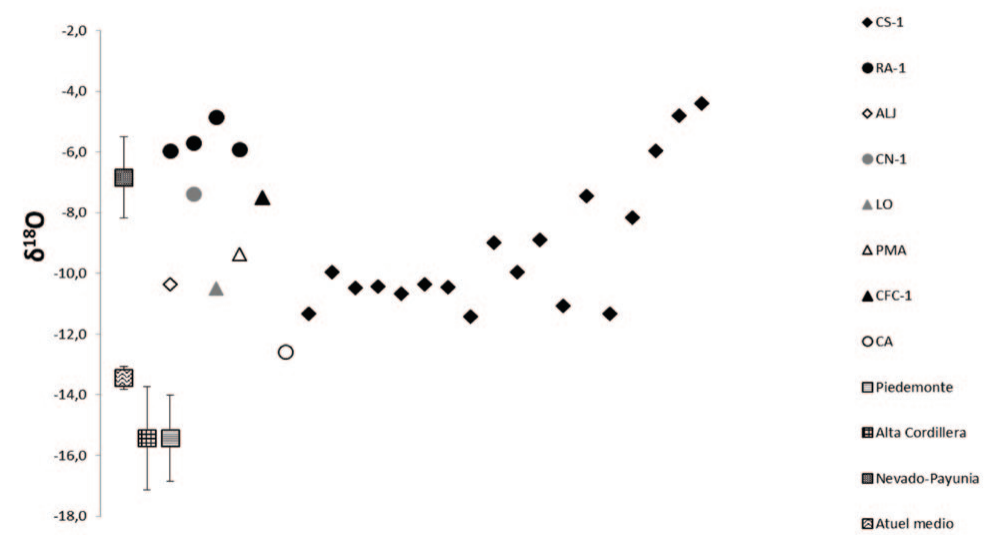

Figura 4. Valores de $\delta^{18} \mathrm{O}$ correspondientes a restos óseos humanos y fuentes de agua del sur de Mendoza. Para las fuentes (Piedemonte, Alta Cordillera, Nevado-Payunia, Atuel Medio) se usaron solo los promedios y desviaciones. Los valores para humanos están graficados individualmente, en color negro=individuos del período pre-1000 años AP, en color gris=individuos del período post-1000 años AP, y en color blanco=individuos sin asignación cronológica.

Figure 4. Values of $\delta^{18} \mathrm{O}$ corresponding to human bone and water sources from southern Mendoza. Average and deviation are used for water sources (Piedemonte, Alta Cordillera, Nevado-Payunia, AtuelMedio). Human values are plotted individually, in black color=individuals with chronologies pre-1000 years $B P$, in grey color=individuals with chronologies post-1000 years BP, and in white color=individuals without chronological assignment. 
explicarse tanto en contextos de caza-recolección como en aquellos con algún componente productivo. Lo mismo sucede con los porcentajes de cambios entésicos en la inserción del bíceps, que es la entesis que resultó más afectada en miembros superiores. En cuanto a la comparación entre conjuntos, CS-1 mostró en líneas generales mayores prevalencias tanto en cambios entésicos como en lesiones articulares, con respecto a los entierros aislados. Sin embargo, las diferencias no son lo suficientemente significativas para inferir un patrón de uso del cuerpo diferencial entre ambos conjuntos, sobre todo considerando que para CS-1 no se logró un buen control de la edad.

Considerando las tendencias observadas, se podría proponer que los marcadores óseos no son concluyentes sobre si el tipo de subsistencia involucró producción de alimentos, aunque podrían corresponderse con grupos que, más allá del tipo de subsistencia puntual, obtenían sus recursos manteniendo una movilidad alta. Los antecedentes de otras regiones señalan similares patrones de estrés en miembros superiores e inferiores tanto para cazadores-recolectores como para agricultores (Bridges 1989, 1991, 1992). Sin embargo, en este contexto transicional, en el que poblaciones altamente móviles habrían incorporado cultígenos a la dieta y probablemente habrían tendido a reducir su movilidad en el marco de un proceso de intensificación en la explotación de los recursos, se esperaba encontrar cambios entésicos y degeneración articular indicativos de este cambio en los patrones de locomoción. Que no se encontraran los patrones esperados puede deberse a que las expectativas iniciales planteadas deben ser reconsideradas y/o a que la reducción de movilidad esperada no tuvo lugar entre estos grupos. La variabilidad de los patrones de estrés ocupacional podría pensarse como el resultado de un contexto dinámico en el que pudieron interactuar estrategias de caza-recolección con algún tipo de estrategia productiva.

En CS-1, que es el sitio con mayor cantidad de individuos, los valores de $\delta^{18} \mathrm{O}$ indican el aprovechamiento de dos grupos de fuentes de agua. La alta variabilidad interindividual es concordante con grupos móviles aprovechando diferentes espacios. RA-1 presenta un patrón más discreto asociado al aprovechamiento de las aguas del área Nevado-Payunia, que se hallan en un rango de 20 a $40 \mathrm{~km}$. CN-1 y CFC-1 se encuentran asociados a Nevado-Payunia, aunque no se encuentran próximos a esta fuente. PMA, ALJ y LO tienen una posición intermedia entre Nevado-Payunia y Atuel Medio, y CA se asocia a Atuel Medio. Este último sitio, se encuentra en las cercanías de la fuente de agua que el individuo estaría usando, por lo que podría suponerse un rango de movilidad menor. En el resto de los casos los valores indicarían un uso promediado de las aguas, por lo que podría considerarse un uso amplio del espacio mediante una estrategia de movilidad alta. Tomando los entierros aislados como un conjunto, el patrón resultante en los valores de $\delta^{18} \mathrm{O}$ es similar al observado en CS-1, por lo que podría esperarse que los individuos de los entierros aislados emplearan estrategias de movilidad similares a los de CS-1.

A pesar de la presencia de cultígenos en el sur de Mendoza desde los 2000 años AP, se infiere de manera preliminar que las poblaciones presentan un patrón de alta movilidad que no coincide con los modelos que sostienen una vida sedentaria para esos momentos. Asimismo, la información sobre la dieta de las poblaciones que habitaron la zona ha ido aumentando en los últimos tiempos (Gil et al. 2010, 2011), indicando contribuciones variables del maíz a la dieta, lo que permite pensar en estrategias posteriores a la incorporación de cultígenos que pueden incluir caza-recolección y aprovechamiento de recursos domesticados, quizás con una producción de cultígenos a pequeña escala. Se destaca que más allá de la presencia de cultígenos y de las diferencias en las modalidades inhumatorias y en la cronología, la estrategia de movilidad alta pudo haberse mantenido.

\section{Conclusiones}

Los datos presentados corresponden a una aproximación inicial en la que se integraron dos líneas de análisis, isótopos de oxígeno y marcadores óseos asociados con actividad, como proxies de la movilidad humana en el sur de Mendoza. Estos datos se insertan en discusiones más amplias vinculadas a procesos dinámicos que habrían afectado a los grupos humanos del centro occidente argentino, como el aumento demográfico, la intensificación en la explotación de recursos y la introducción de cultígenos. Los cambios entésicos y las lesiones degenerativas articulares no fueron concluyentes acerca de la estrategia de movilidad, aunque las altas prevalencias de ambos marcadores podría estar asociada a una movilidad alta, considerando además la información isotópica que indica el uso de diferentes áreas. La información obtenida requiere una profundización temporal y espacial que permita ampliar las tendencias. Los objetivos en el corto plazo serán incluir en el análisis sitios del valle del Atuel que presentan características similares a las de CS-1 en cuanto al $n$ y modalidad de inhumación, y realizar fechados para aquellos sitios analizados que aún no poseen asignación cronológica. Asimismo, para poder discutir división sexual del trabajo y patrones diferenciales en el uso del cuerpo entre masculinos y femeninos, se buscará incluir muestras con un mayor número de individuos femeninos y se aplicarán métricas de huesos largos para estimar el sexo en los enterratorios múltiples mezclados.

San Rafael, 30 de Marzo 2017

\section{Agradecimientos}

Este trabajo se realizó en el marco de los proyectos PICT 2012-1015 y PICT 2013-0881. Especiales agradecimientos 
para los Dres. Adolfo Gil, Gustavo Neme y Leandro Luna, cuyos conocimientos y experiencia hicieron este trabajo posible. Gracias al equipo de investigación del Depto. de Antropología del Museo de Historia Natural de San Rafael y del IANIGLA-Grupo Vinculado San Rafael por motorizar las investigaciones arqueológicas del sur de Mendoza y otras áreas.

\section{Bibliografía}

Al-Oumaoui, I., S. Jiménez-Brobeil, y P. du Souich. 2004. Markers of Activity Patterns in some Populations of the Iberian Peninsula. International Journal of Osteoarchaeology 14: 343-359.

Bridges, P. S. 1989. Changes in activity with the shift to agriculture in the southeastern United States. Current Anthropology 30: 385-394.

Bridges, P. S. 1992. Prehistoric arthritis in the Americas. Annual Review of Anthropology 21: 67-91.

Bridges, P. S. 1994. Vertebral arthritis and physical activities in the prehistoric Southeastern United States. American Journal of Physical Anthropology 93: 83-93.

Bruzeck, J. 2002. A method for visual determination of sex, using the human hip bone. American Journal of Physical Anthropology 117: 157-168.

Buikstra, J. E. y D. H. Ubelaker. 1994. Standards for data collection from human skeletal remains. Arkansas Archaeological Survey Research Series N 44, Arkansas.

Cantarella, V. 1999. Bones and muscles: an illustrated anatomy. Wolf Fly Press, South Westerlo, New York.

Capasso, L., K. Kennedy y C. Wilczak. 1999. Atlas of occupational markers on human remains. Edigrafital S.P.A., Teramo.

Cardoso, F. A., C. Y. Henderson. 2013. The Categorisation of Occupation in Identified Skeletal Collections: A Source of Bias? International Journal of Osteoarchaeology 23: 186-196.

Castro, V., M. Tarragó. 1992. Los inicios de la producción de alimentos en el Cono sur de América. Revista de Arqueología Americana 6: 91-124.

Cope, J. M., A. C. Berryman, D. L. Martin, y D. D. Potts. 2005. Robusticity y Osteoarthritis at the Trapeziometacarpal Joint in a Bronze Age Population From Tell Abraq, United Arab Emirates. American Journal of Physical Anthropology 126: 391-400.

Dansgaard, W. 1964. Stable isotopes in precipitation. Tellus 16(4): 436-468.
Galtés, I., X. Jordana, C. García y A. Malgosa. 2007. Marcadores de Actividad en Restos óseos. Cuadernos Médicos Forenses 13: 179-189.

Gat, J. R. 1996. Oxygen and hydrogen isotopes in the hydrologic cycle. Annual Review of Earth and Planetary Sciences 24: 225-262.

Giardina, M. A., M. Corbat, E. A. Peralta, G. Cochero, F. Franchetti, y L. Salgán. 2015. El registro arqueológico en sitio La Olla (San Rafael, Mendoza): implicaciones para las ocupaciones humanas en el valle medio del río Atuel. Revista del Museo de Antropología 8(1): 51-66.

Gil, A. F. 1997-1998. Cultígenos prehispánicos en el Sur de Mendoza. Discusión en torno al límite meridional de la agricultura andina. Relaciones 22-23: 295-318.

Gil, A. F. 2003. Zea mays on the South American Periphery: Chronology and Dietary Importance. Current Anthropology 44(2): 295-300.

Gil, A. F., G. A. Neme. 2010. Registro arqueológico en la cuenca media del Atuel: viejos y nuevos problemas; viejos y nuevos datos. En: Zárate, M., A. Gil y G. Neme (comps.), Condiciones paleoambientales y ocupaciones humanas durante la transición Pleistoceno-Holoceno y Holoceno de Mendoza, pp. 239-275. Sociedad Argentina de Antropología, Buenos Aires.

Gil, A. F., R. H. Tykot, G. A. Neme, y N. R. Shelnut. 2006. Maize on the frontier: isotopic and macrobotanical data from central-western Argentina. En: Staller, J., R. Tykot, y B. Benz (eds.), Histories of Maize Multidisciplinary Approaches to the Prehistory, Biogeography, Domestication, and Evolution of Maize, pp. 199-214. Academic Press, Burlington.

Gil, A. F., G. A. Neme, y R. H. Tykot. 2010. Isótopos estables y consumo de maíz en el Centro Occidente Argentino: tendencias temporales y espaciales. Chungara 42(2): 497-513.

Gil, A. F., G. A. Neme, y R. H. Tykot. 2011. Stable Isotopes and Human Diet in Central Western Argentina. Journal of Archaeological Science 38: 1395-1404.

Gil, A. F., G. A. Neme, A. Ugan, y R. H. Tykot. 2012. Oxygen isotopes and human residential mobility in central western Argentina. International Journal of Osteoarchaeology DOI: 10.1002/oa.1304

Hammer, Ø., D. A. Harper y P. D. Ryan. 2001. PAST: Paleontological Statistics Software Package for Education and Data Analysis. Paleontología Electrónica 4(1): 9.

Hawkey, D., C. Merbs. 1995. Activity-Induced Musculoskeletal Stress Markers (MSM) and Subsistence 
Strategy Changes among Hudson Bay Eskimos. International Journal of Osteoarchaeology 5: 324-338.

Henderson, C. Y., D. D. Craps, A. C. Caffell, A. R. Millard, y R. Gowland. 2013b. Occupational Mobility in 19th Century Rural England: The Interpretation of Entheseal Changes. International Journal of Osteoarchaeology 23: $197-210$.

Henderson, C. Y., V. Mariotti, D. Pany-Kucera, S. Villotte, y C. Wilczak. 2013a. Recording specific entheseal changes of fibrocartilaginous entheses: initial tests using the Coimbra method. International Journal of Osteoarchaeology 23: 152-162.

Henderson, C. Y., V. Mariotti, D. Pany-Kucera, S. Villotte, C. Wilczak. 2016. The new "Coimbra method": a biologically appropriate method for recording specific features of fibrocartilaginous entheseal changes. International Journal of Osteoarchaeology 26(5): 925-932.

Jurmain, R. 1977. Stress and Etiology of Osteoarthritis. American Journal of Physical Anthropology 46: 353-366.

Jurmain, R., F. Alves Cardoso, C. Henderson y S. Villotte. 2012. Bioarchaeology's Holy Grail. The Reconstruction of Activity. En: Al Grauer (ed.), A Companion to Paleopathology, pp. 531-552. Wiley - Blackell, Nueva York.

Kennedy, K. 1989. Skeletal markers of occupational stress. En: D. M. Yasar Iscan y K. Kennedy (eds.), Reconstruction of Life from the Skeleton, pp. 129-160. Alan R. Liss, Nueva York.

Knudson, K. J. 2009. Oxygen isotope analysis in a land of environmental extremes: the complexities of isotopic work in the Andes. International Journal of Osteoarchaeology 19(2): 171-191.

Lagiglia, H. A. 1977. "Arqueología y Ambiente Natural de los Valles del Atuel y Diamante". Facultad de Ciencias Naturales y Museo, Universidad Nacional de La Plata, Argentina. Tesis de postgrado. Universidad Nacional de La Plata.

Lagiglia, H. A. 1981. El proceso de agriculturización del Sur de Cuyo: la Cultura del Atuel II. En: Actas del V Congreso Nacional de Arqueología Argentina 1: 231252. San Juan.

Lovejoy C. O, R. S Meindl, T. R. Pryzbeck y R P. Mensforth. 1985. Chronological metamorphosis of the auricular surface of the ilium. A New method for the determination of adult skeletal age at death. American Journal Physical Anthropology 68(1): 15-28.

Luna, L., C. Aranda y A. Amorim Alves. 2017. Reflexiones sobre el relevamiento y análisis comparativo de patologías osteoarticulares en restos esqueletales humanos. Revista Argentina de Antropología Biológica 19(1): 1-8.

Meindl, R. S., C. O. Lovejoy. 1989. Age changed in the pelvis: Implications for paleodemography. En M. Y. Iscan (ed.), Age Markers in the human skeleton, pp. 137-168. CC. Thomas Pub., Springfield, Illinois.

Mendoça De Souza, S. 1992. Traumatismos vertebrais como indicadores de atividade fisica na populaçao da Furna do Estrago, Pernambuco, Brasil. En: L. F. Ferreira y A. J. de Araújo (eds.), Paleopatologia e PaleoepidemiologiaEstudos Multidisciplinares, pp. 123-140. Panorama ENSP, Río de Janeiro.

Neme, G. A. 2007. Cazadores-recolectores de altura en los Andes meridionales: el alto valle del río Atuel. British Archaeological Reports International Series 1591 Archaeopress. Oxford.

Neme, G. A., A. F. Gil. 2008. Biogeografía humana en los Andes Meridionales: tendencias arqueológicas en el Sur de Mendoza. Chungara 40: 5-18.

Neme, G. A., A. F. Gil. 2012. El registro arqueológico del sur de Mendoza en perspectiva biogeográfica. En: A. Gil y G. Neme (comps.), Paleoecología humana en el sur de Mendoza: perspectivas arqueológicas, pp. 255-279. Sociedad Argentina de Antropología, Buenos Aires.

Neves, W. 1984. Estilo de Vida e osteobigrafia: a reconstituição do comportamento pelos osso humanos. Revista Pré-História 6: 287-291.

Peralta, E. A. 2015. "Subsistencia y movilidad en la cuenca media del Atuel en el Holoceno Tardío: una perspectiva bioarqueológica, aplicando el análisis de marcadores de estrés ocupacional". Facultad de Filosofía y Letras, Universidad de Buenos Aires, Argentina. Tesis de grado. Universidad de Buenos Aires.

Quevedo, S. 2000. Patrones de actividad a través de las patologías en poblaciones arcaicas de puntas Teatinos del norte semiárido Chileno. Chungara 32(1): 7-9.

Robb J. 1998. The interpretation of skeletal muscle site: an statistical approach. International Journal of Osteoarchaeology 8: 363-377.

Robson Brown K., P. Pollintine y M. A. Adams. 2008. Biomechanical implications of degenerative joint disease in the apophyseal joints of human thoracic and lumbar vertebrae. American Journal of Physical Anthropology 136: 318-326.

Rodrigues Carvalho, C. 2004. "Marcadores de Estresse Ocupacional em Populações Sambaquieiras do Litoral 
Fluminense". Fundación Osvaldo Cruz, Rio de Janeiro, Brasil. Tesis de postgrado. Fundación Osvaldo Cruz.

Salega S. y M. Fabra. 2013. Niveles de actividad física en poblaciones de las sierras y las llanuras de la provincia de Córdoba (Argentina) durante el Holoceno tardío. Relaciones 38: 401-420.

Santos, A. L., F. Alves-Cardoso, S. Assis y S. Villotte. 2011. The Coimbra Workshop in Musculoskeletal Stress Markers (MSM): an annotated review. Antropologia Portuguesa 28: 135-161.

Scabuzzo, C. 2012. Estudios bioarqueológicos de Marcadores de Estrés Ocupacional en cazadores recolectores pampeanos del Holoceno temprano-medio. Análisis de la serie esqueletal de Arroyo Seco 2. Revista Argentina de Antropología Biológica 14(1): 17-31.

Semper, J. y H. A. Lagiglia. 1962-1968. Arqueología de la Gruta del Indio. Revista Científica de Investigaciones 1(4): 89-158.

Sponheimer, M., J. A. Lee-Thorp. 1999. Oxygen isotopes in enamel carbonate and their ecological significance. Journal of Archaeological Science 26(6): 723-728.

Todd, T. W. 1920. Age changes in the pubic bone: The white male pubis. American Journal of Physical Anthropology 3: 427-470.

Turner, B. L., G. D. Kamenov, J. D. Kingston, y G. J. Armelagos. 2009. Insights into immigration and social class at Machu Picchu, Peru based on oxygen, strontium, and lead isotopic analysis. Journal of Archaeological Science 36(2): 317-332.
Ugan, A., G. A. Neme, A. F. Gil, J. Coltrain, R. H. Tykot, y P. S. Novellino. 2012. Geographic variation in bone carbonate and water $\delta^{18} \mathrm{O}$ values in Mendoza, Argentina and their relationship to prehistoric economy and settlement. Journal of Archaeological Science 39: 27522763.

Villotte, S., C. J. Knüsel. S. 2013. Understanding Entheseal Changes: Definition and Life Course Changes. International Journal of Osteoarchaeology 23: 135-146.

Villotte, S., S. E. Churchill, O. J. Dutour, y D. HenryGambier. 2010b. Subsistence activities and the sexual division of labor in the European Upper Paleolithic and Mesolithic: Evidence from upper limb enthesopathies. Journal of Human Evolution 59: 35-43.

Villotte, S., D. Castex, V. Couallier, O. Dutour, C. J. Knüsel, y D. Henry-Gambier. 2010a. Enthesopathies as Occupational Stress Markers: Evidence From the Upper Limb. American Journal of Physical Anthropology 142: 224-234.

Weiss, E., R. Jurmain. 2007. Osteoarthritis Revisited: A Contemporary Review of Aetiology. International Journal of Osteoarchaeology 17: 437-450

White, C., F. J. Longstaffe, y K. R. Law. 2004. Exploring the effects of environment, physiology and diet on oxygen isotope ratios in ancient Nubian bones and teeth. Journal of Archaeological Science 31(2): 233-250.

Wilczak, C. A. 1998. Consideration of Sexual Dimorphism, Age, and Asymmetry in Quantitative Measurements of Muscle Insertion Sites. International Journal of Osteoarchaeology 8: 311-325. 